\title{
Duloxetine for the management of fibromyalgia syndrome
}

\author{
This article was published in the following Dove Press journal: \\ Journal of Pain Research \\ 16 July 2009 \\ Number of times this article has been viewed
}

\author{
Beth A Scholz \\ Cara L Hammonds \\ Chad S Boomershine \\ Department of Medicine, Vanderbilt \\ University, Nashville, TN, USA
}

Correspondence: Chad S Boomershine Department of Medicine, Vanderbilt University, T3219 MCN, II6I

2 Ist Ave South, Nashville,

TN 37232-268I, USA

$\mathrm{Tel}+\mathrm{I}$ 615-322-4746

Fax + | 615-322-6248

Email chad.boomershine@vanderbilt.edu

\begin{abstract}
Fibromyalgia syndrome (FMS) is a widespread pain condition associated with a wide range of additional symptoms including fatigue, insomnia, depression, anxiety and stiffness. Duloxetine is one of three medications currently FDA approved for use in FMS management. Duloxetine is a mixed serotonin and norepinephrine reuptake inhibitor (SNRI) that functions by increasing central nervous system levels of serotonin and norepinephrine. This review is a primer on use of duloxetine in FMS management and includes information on pharmacology and pharmacokinetics, a review of the three duloxetine FMS treatment trials currently in publication, a discussion of the safety and tolerability of duloxetine, and patientfocused perspectives on duloxetine use in FMS management. Duloxetine has proven efficacy in managing pain and mood symptoms in adult FMS patients with and without major depressive disorder. However, due to side effects, duloxetine must be used with caution in patients with fatigue, insomnia, gastrointestinal complaints, headache, cardiovascular disease, bleeding-risk, and in those 24 years of age and younger due to risk of suicidality. Duloxetine use should be avoided in patients with liver disease or alcoholics. As with all medications, duloxetine is best used as part of an individualized regimen that includes nonpharmacologic modalities of exercise, education and behavioral therapies.
\end{abstract}

Keywords: fibromyalgia, duloxetine, SNRI, safety

\section{Introduction to fibromyalgia management}

Fibromyalgia syndrome (FMS) is a disorder of chronic widespread pain (CWP) and tenderness associated with a wide range of other symptoms including fatigue, nonrestorative sleep, depression, anxiety, and stiffness. ${ }^{1}$ FMS is a common disorder, with published prevalence rates ranging from $0.5 \%$ to $5 \%$ in countries across the world. ${ }^{2}$ However, these prevalence rates underestimate the true prevalence of the disorder as they are based on fulfillment of ACR tender point classification criteria that fail to identify nearly $50 \%$ of FMS patients, particularly in males who usually have fewer tender points. ${ }^{3}$ Despite the high prevalence of FMS, some clinicians have been reluctant to diagnose and treat the disorder based on a belief that the condition is a psychogenic disorder with no physiologic basis resulting from the stresses of modern life, the feeling that acknowledgement of FMS worsens patient health by reinforcing maladaptive coping behaviors and an impression that FMS patients do not improve with treatment. ${ }^{4}$ However, there is evidence that FMS has existed for centuries and is prevalent in societies that experience none of the stresses of modernity. ${ }^{5,6}$ Also, objective evidence utilizing numerous modalities has linked FMS to genetic polymorphisms in the serotonergic, dopaminergic and catecholaminergic systems of pain transmission and 
processing that result in multiple neurochemical abnormalities including deficient CNS concentrations of serotonin (5-HT) and norephinephrine (NE). ${ }^{7,8}$ Work by Taieb and colleagues has shown providing patients with a FMS diagnosis improves the health of both patients and the medical system by reducing medication use as well as the number of office visits and referrals. ${ }^{9}$ Further evidence for the improvement in FMS symptoms with treatment comes from numerous positive therapeutic trials and FDA approval of three medications for FMS management including duloxetine, milnacipran and pregabalin because of their efficacy in improving FMS pain and global symptom burden.

While all FMS patients have CWP and tenderness, individual patients differ widely in the number and severity of other symptoms they experience. This symptom heterogeneity requires that each FMS patient be individually assessed to identify problematic symptoms and an individualized therapeutic regimen be developed to manage each symptom. This approach limits treatment failures that often arise from an exclusive focus on pain and has been recommended by multiple professional societies including the European League Against Rheumatism (EULAR). ${ }^{10}$ However, a symptom-based approach is hampered by the inability of many patients to effectively articulate their symptom burden and the difficulty of clinicians to interpret patient complaints into a coherent intellectual framework from which to develop a treatment plan. While self-report questionnaires to quantify the severity of numerous symptoms exist, their length and complexity often preclude use in busy clinical practice settings. To overcome these difficulties, one of the authors (CSB) has developed the modified visual analogue scale fibromyalgia impact questionnaire (mVASFIQ). ${ }^{11,12}$ The mVASFIQ utilizes 7 visual analogue scales (VASs) from a disease-neutral fibromyalgia impact questionnaire (FIQ) in combination with the FIBRO mnemonic to assess symptoms of Fatigue (tiredness), Insomnia (sleep quality), Blues (mood disorders including depression and anxiety), Rigidity (stiffness) and Ow! (for pain and work difficulty). This scale provides a simplified global measure of disease severity along with individual symptom subscales that can be used to individualize therapeutic regimens and monitor treatment response.

While a thorough review of FMS management is beyond the scope of the current work, many excellent FMS treatment reviews exist. ${ }^{10,11,13}$ Three general points should be kept in mind when treating FMS patients. First, primary disorders that mimic FMS must be ruled out before symptomatic therapies are used including vitamin deficiencies, anemia, and metabolic, oncologic or inflammatory disorders. Second, since over $50 \%$ of FMS patients suffer from multiple medication intolerances, ${ }^{14}$ medications should be started individually at low dose and slowly up-titrated and/or combined. Also, multiple medications or combinations may need to be tried before finding a regimen the patient will tolerate. Finally, while this review focuses on pharmacologic treatment with duloxetine, medications have a limited role in FMS management. Medications provide symptom relief so that patients can participate in nonpharmacologic modalities that provide long-term disease coping strategies. ${ }^{10}$ Nonpharmacologic treatments with proven efficacy in managing FMS involve a combination of aerobic and resistance exercise, education, and cognitive behavioral therapies. ${ }^{10}$

\section{Review of pharmacology, mode of action, and pharmacokinetics of duloxetine}

Duloxetine $\mathrm{HCl}$ is a mixed serotonin and norepinephrine reuptake inhibitor (SNRI), meaning that it acts to increase levels of both serotonin and norepinephrine (NE) in the central nervous system (CNS). ${ }^{15}$ This mechanism of activity has been verified by measurement of platelet serotonin (5-HT) levels and urinary excretion of $\mathrm{NE}$ and its metabolites as markers of circulating 5-HT and NE. ${ }^{16}$ Duloxetine is one of a group of four FDA approved SNRIs that also includes desvenlafaxine, milnacipran and venlafaxine. Although medications in other antidepressant classes, such as the tricyclic antidepressants (TCAs), monoamine oxidase inhibitors (MAOIs) and high doses of the first-generation selective serotonin reuptake inhibitors (SSRIs), can also increase both 5-HT and NE levels in the CNS, the SNRIs have been shown to be safer and better tolerated. ${ }^{17}$ While all SNRIs increase both 5-HT and NE levels, they differ in their ratio of selectivity for 5-HT versus NE reuptake inhibition (Table 1). ${ }^{18,19}$ In vitro data shows that duloxetine, with a 5-HT:NE reuptake inhibition ratio of 10:1, is intermediate among the SNRIs. While differences in 5-HT:NE ratios may affect the relative efficacy of the SNRIs in treating FMS symptoms, the clinical significance of this difference is currently unknown and will require headto-head treatment trials. However, it should be noted that all SNRIs have 5-HT:NE ratios much more neutral than the SSRIs which have ratios between 100 and 2600:1. ${ }^{20}$

Duloxetine is metabolized mainly through the hepatic cytochrome P450 (CYP450) system. ${ }^{21}$ Because of its extensive hepatic metabolism, duloxetine should ordinarily not be prescribed to patients with substantial alcohol use or 
Table I Relative 5-HT:NE in-vitro reuptake inhibition of SNRI-active drugs

\begin{tabular}{ll}
\hline Drug name & $\begin{array}{l}\text { 5-HT:NE reuptake } \\
\text { inhibition ratio }\end{array}$ \\
\hline Venlafaxine & $30: 1^{18}$ \\
Duloxetine & $10: 1^{18}$ \\
Desvenlafaxine & $10: 1^{19}$ \\
Amitriptyline & $8: 1^{58}$ \\
Milnacipran & $1: 1^{18}$ \\
\hline
\end{tabular}

Abbreviations: 5-HT, serotonin; NE, norepinephrine; SNRI, serotonin and norepinephrine reuptake inhibitor.

evidence of chronic liver disease. Smoking lowers serum concentrations of many psychotropic medications through its effects on CYP1A2, and this causative effect has been implicated in a $30 \%$ reduction from expected serum concentrations in smokers taking duloxetine. ${ }^{22}$ However, the clinical relevance of this altered metabolism in terms of the need for dose adjustment remains unclear. The half-life of duloxetine in plasma is 12.5 hours and, as with many SSRIs, this relatively short half-life presents a risk of withdrawal syndrome if the drug is stopped abruptly. ${ }^{15,21}$ Duloxetine clearance decreases with age but not to a degree requiring dose adjustment, and gender has no significant effect on half-life. ${ }^{23}$ Taking duloxetine with meals prolongs the time to peak plasma concentration by 6 to 10 hours but does not alter the peak plasma concentration..$^{21}$ The prolongation of time to peak plasma concentration by taking duloxetine with a meal can limit the development of side effects and it is generally recommended duloxetine be taken with food. However, crushing capsules or otherwise removing the enteric coating (such as sprinkling in food) can lead to erratic absorption of duloxetine and should be avoided. ${ }^{23}$ Lilly Research Laboratories, the manufacturer of duloxetine, has investigated the effects of mixing whole capsules in food products commonly used as delivery agents and found that while applesauce and apple juice had no effect on enteric capsule integrity, chocolate pudding hastened the dissolution of duloxetine. ${ }^{24}$

\section{Duloxetine efficacy studies}

Three randomized, double-blind, placebo-controlled trials (RCTs) of duloxetine in the treatment of FMS have been published to date. ${ }^{25-27}$ The first was a 12-week trial that examined the effect of duloxetine at a dose of $60 \mathrm{mg}$ twice daily compared with placebo in male and female FMS patients with and without major depressive disorder (MDD). ${ }^{25}$ Subjects were recruited through 5 academic centers and 13 "independent research centers" within the US.
Trial subjects were required to meet ACR classification criteria for fibromyalgia including chronic widespread pain for $\geq 3$ months and the presence of at least 11 of 18 tender points. ${ }^{1}$ Exclusion criteria included comorbid psychiatric illness other than MDD, treatment-refractory FMS, and pending disability review. Fibromyalgia Impact Questionnaire (FIQ) global and individual FIQ VAS pain severity scores were the coprimary endpoints. The FIQ is the most widely used measure for quantifying the severity of FMS symptoms and includes 20 questions that assess functionality with activities of daily living (ADL), work difficulty, general feelings of well-being, sleep quality and the severity of symptoms including pain, fatigue, depression, anxiety, and stiffness. ${ }^{28}$ Secondary endpoints included individual FIQ component scores as well as scores on the short form Brief Pain Inventory, ${ }^{29}$ Clinical Global Impression of Severity (CGI) (a scale measuring the clinician's impression of change in disease severity ranging from 1 [normal, not at all ill] to 7 [among the most extremely ill patients]), ${ }^{30}$ the Patient Global Impression of Improvement scale (PGI) (a scale measuring patient response to therapy ranging from 1 [very much better] to 7 [very much worse]), ${ }^{30}$ the Beck Depression and Anxiety Inventories (21-question survey of depression or anxiety symptoms, respectively), ${ }^{31,32}$ the Medical Outcomes Study Short Form 36 (SF-36), Quality of Life in Depression Scale, and the Sheehan Disability Scale (SDS). The SF-36 contains both physical and mental components to assess global patient function. ${ }^{33}$ The SDS assesses functional impairment through use of home, school/work, and social domains. ${ }^{34}$ Eighty-nine percent of the subjects in the study were women and $87 \%$ were racially classified as white.

While both FIQ global and VAS pain scores showed statistically significant improvement in the duloxetine treatment group compared with placebo after 4 weeks, this improvement was not maintained and, despite significant improvement in FIQ global scores occurring in the duloxetinetreated group at the 12-week time point, the study failed to reach its coprimary endpoint due to a lack of demonstrated improvement in VAS pain scores at 12 weeks. Since the study did not meet its coprimary endpoints, assessment of secondary endpoints are not valid. However, the authors reported analyses that provide insights for use of duloxetine in treating FMS. Most interesting was the finding that no statistically significant improvement was noted in any of the primary or secondary endpoints in male study participants. While this lack of improvement may have been due to the limited number of male subjects enrolled in the study (23 in total), gender differences in medication response cannot be ruled out. 
Also, the low numbers of male participants highlight the difficulties inherent in enrolling male subjects in FMS studies since the current ACR classification criteria create a 9:1 bias in women:men that is much higher than the 4:1 female:male ratio seen in our medical center (unpublished results determined from a search of electronic medical records utilizing the Vanderbilt University Record Counter). The female bias associated with the ACR criteria is created by the higher number of tender points seen in females. ${ }^{35}$ Male FMS studies will likely have to await more gender-neutral revisions of the ACR FMS criteria that are currently under development. Female subjects treated with duloxetine experienced significant improvements as determined by decreased number of tender points, increased pain threshold, and improvements in CGI-Severity, PGI-Improvement, and BPI scores. However, because of the high prevalence of mood symptoms in FMS patients and the known association between mood and pain, it has been difficult in previous studies to determine whether medications are improving pain symptoms due to their effect on mood or whether they are improving pain symptoms directly. To test the direct effect of duloxetine on pain reduction, a path analysis technique was performed utilizing three regression models to compare the response to mood symptoms (both anxiety and depression) utilizing the Beck Inventories to the response to pain that allowed estimation of the percentage of direct and indirect effects on the total treatment effect. ${ }^{36}$ The path analysis showed duloxetine had a $61 \%$ to $83 \%$ direct effect on pain improvement (depending on which pain scale was being evaluated) that was independent of improvement in mood symptoms. This finding indicates duloxetine may provide pain relief in FMS patients without coexisting mood symptoms. However, this study has numerous shortcomings limiting its applicability. Duloxetine dose amount and administration in the study differed from the currently indicated dosing regimen for FMS. In the trial, patients were placed on a forced dose titration scheme of $20 \mathrm{mg}$ once daily for 5 days, $20 \mathrm{mg}$ twice daily for 3 days, $40 \mathrm{mg}$ twice daily for 2 days and $60 \mathrm{mg}$ twice daily thereafter. This differs from the recommended dosing regimen which is $30 \mathrm{mg}$ once daily for 1 week followed by $60 \mathrm{mg}$ once daily if patient fails to respond. ${ }^{37}$ Also, FIBRO symptoms such as Fatigue and Insomnia were not studied despite both fatigue and insomnia being frequently reported treatment-emergent adverse events (TEAEs) with duloxetine use in patients with MDD. ${ }^{38}$

A later 12-week duloxetine RCT studying the effect of duloxetine treatment in 354 female FMS patients with and without MDD addressed the limitations of the first study and provided more clinically applicable conclusions. ${ }^{26}$ As with the first trial, the majority of patients were Caucasian (89.5\%), with $8.2 \%$ classified as 'Hispanic' and $2 \%$ being of 'African descent.' Patients were randomized to receive placebo or duloxetine $60 \mathrm{mg}$ once or twice daily. The study had a single primary outcome measurement which was change in 24 hour average pain severity as scored by the BPI VAS. ${ }^{29}$ A $30 \%$ improvement from baseline was considered a significant response, and multiple secondary endpoints were determined including worst and least pain in BPI component scores, FIQ global, and mean tender point threshold as measured by Fischer dolorimetry. Twenty-six percent of enrollees had comorbid MDD, and several scales were included as surrogate markers for improvement in depression and for use as secondary endpoints including the Hamilton Depression rating scale $\left(\mathrm{HAMD}_{17}\right)$, Quality of Life in Depression Scale, SF-36, and SDS. The HAMD ${ }_{17}$ is a clinician-rated scale that includes 17 questions covering mood, sleep, and somatic complaints. ${ }^{39}$ In the trial, both active treatment groups met the primary study endpoint with significant improvement in pain severity of $37.5 \%$ from baseline compared with an $18.5 \%$ improvement in the placebo group. The differences between both active treatment groups and placebo were statistically significant, but there was no difference between the once and twice daily duloxetine treatment groups. The same pattern of patient response was noted with regard to the FIQ; as both duloxetine groups improved to a similar statistically significant degree compared with placebo and no significant difference was seen between the once- and twice-daily active treatment groups. Patients treated with duloxetine at $60 \mathrm{mg}$ twice daily showed a statistically significant improvement in tender point threshold not seen in the once daily or placebo treated groups. However, improvement in tender point threshold is a controversial FMS outcome measure since tender point threshold measurements are difficult to standardize and reproduce consistently, ${ }^{40}$ pain threshold is influenced by patient-independent factors, ${ }^{41}$ and weak to no associations have been found between tender point pain ratings and FMS symptoms. ${ }^{42}$ Overall, the study found no significant difference between patients treated with duloxetine once or twice daily in any of the other measured primary or secondary endpoints. This finding led the authors to conclude that the $60 \mathrm{mg}$ twice daily dose provided no additional benefit and may expose patients to dose-dependent adverse events without improved efficacy, substantiated by the current recommended dosing for FMS patients. ${ }^{37}$ The symptom improvement with duloxetine treatment was rapid, with significant improvements in both pain and FIQ 
global scores seen within 1 week and reaching a maximum within 4 to 6 weeks. However, patients in the trial were immediately placed on a daily duloxetine dose of $60 \mathrm{mg}$ and the recommended dose escalation protocol using $30 \mathrm{mg}$ once daily for 1 week was not used, ${ }^{37}$ so this rapid response may not be seen in routine clinical practice. Consistent with the initial duloxetine RCT, Path analysis showed 75.6\% and $86.9 \%$ of the improvement in pain symptoms was due to a direct effect of duloxetine on pain at doses of $60 \mathrm{mg}$ once and twice daily, respectively, that was independent of improvement in depressive symptoms. Further evidence for a direct role of duloxetine in providing pain relief was provided by data showing patients with and without baseline MDD had similar improvements in pain response.

Russell et al published a third RCT to assess the efficacy and safety of duloxetine at doses of 20,60 and $120 \mathrm{mg}$ once daily. ${ }^{27}$ In this study, 520 patients meeting ACR criteria for FMS with or without current MDD were treated for 6 months. The majority of participants were female (94.8\%), white $(84.2 \%)$, and averaged 51 years of age. This study used a more standard dosing escalation strategy, with patients in the $60 \mathrm{mg}$ duloxetine group given $30 \mathrm{mg}$ once daily for the first week and patients in the $120 \mathrm{mg}$ group given $30 \mathrm{mg}$ once daily for 1 week and then $60 \mathrm{mg}$ daily for 1 week before being placed on $120 \mathrm{mg}$ once daily for the remainder of the trial. While the trial evaluated patients over 6 months, it was actually a 3-month study with a 3-month extension phase since the coprimary endpoints of BPI average pain severity and PGI-I scores were evaluated at 3 months and the $20 \mathrm{mg}$ /day duloxetine group had their dosing increased to $60 \mathrm{mg}$ once daily after the initial 3 months. Secondary endpoints included BPI and PGI-I scores at 6 months, FIQ global, CGI-S, tender-point pain assessments using Fischer dolorimetry, the Multidimensional Fatigue Inventory (MFI), ${ }^{43} \mathrm{HAMD}_{17}$, Quality of Life in Depression Scale, SF-36 and SDS. Twenty-four percent of study participants had comorbid MDD at baseline to determine the effect that duloxetine treatment had on this subpopulation. As previously discussed, the inclusion of FMS patients with MDD is important since approximately $30 \%$ of FMS patients have MDD. ${ }^{2}$ The trial met its primary endpoints, with patients in both duloxetine $60 \mathrm{mg} /$ day and $120 \mathrm{mg}$ /day treatment groups demonstrating statistically greater reductions in pain severity and improvements in PGI-I scores after both 3 and 6 months of treatment compared with placebo. While PGI-I scores showed statistical improvement in the $20 \mathrm{mg}$ /day duloxetine group, this group failed to meet the coprimary endpoint of pain reduction. However, the degree of pain reduction was similar to that seen in the $60 \mathrm{mg} /$ day group. The lack of statistical significance was likely related to a lower statistical power in the $20 \mathrm{mg}$ /day group due to $50 \%$ fewer patients in this group and not due to lack of efficacy in pain relief. This finding, along with findings of similar efficacy in improving FIQ global scores, an equally rapid statistically significant improvement in pain and PGI-I scores, and a lower incidence of TEAEs, indicates serious consideration should be made for beginning all FMS patients on a $20 \mathrm{mg}$ once daily dose of duloxetine and assessing treatment response before dose escalations are made. The fact that all groups showed significant improvement after one week, during which time all 3 groups were receiving either 20 or $30 \mathrm{mg}$ of duloxetine once daily, lends further support for the efficacy of lower duloxetine doses. In contrast to the first duloxetine RCT, ${ }^{25}$ the Russell et al study showed male and female patients treated with duloxetine had similar improvement in the average pain severity after both 3 months and 6 months of treatment. Global improvement, as assessed by both patient (PGI-I) and clinician (CGI-S) measures was significantly improved after both 3 and 6 month time points in duloxetine-treated groups as compared with placebo (except for the 6 month time point for the PGI-I in the $60 \mathrm{mg}$ /day group and the CGI-S for the $20 \mathrm{mg}$ /day group at 3 months). The FIQ score was also significantly improved at 3 months in all three duloxetine groups compared to placebo, but the improvement was lost at 6 months. In contrast to the second duloxetine FMS RCT, ${ }^{26}$ no improvement was seen in mean tender-point threshold for any active treatment group compared with placebo.

Consistent with both previous discussed studies, ${ }^{25,26}$ duloxetine-treated patients experienced similar improvement in pain regardless of the presence or absence of MDD, and the majority of duloxetine effect on pain was directly mediated and not due to improvement in depressive symptoms (from 62.2 to $82.3 \%$ depending on assessment measure and dose). Surprisingly, only patients with pre-existing MDD treated with $120 \mathrm{mg}$ /day of duloxetine showed improvement in depressive symptoms after 3 months as assessed by $\mathrm{HAMD}_{17}$ scores, and no duloxetine dose revealed a clinically significant antidepressant effect at 6 months. In contrast to the two previously discussed studies, the Russell et al study assessed the effect of duloxetine on fatigue symptoms. Duloxetine treatment showed similar numeric improvement in MFI general fatigue scores at 3 and 6 month end-points for all doses, but this improvement was no better than that seen with placebo. However, when MFI sub-scores were analyzed, patients in all 3 duloxetine treatment groups 
showed statistically significant improvements in the mental fatigue domain (which assesses attention and concentration) at the 6 month time point compared with placebo, indicating duloxetine may be beneficial in patients with "fibrofog."

It is important to note that while "treatment refractory" patients were excluded from all of the duloxetine RCTs, this patient category was not explicitly defined. From a clinical perspective, one could consider patients who have not experienced improvement on multiple medications to be refractory, and this proportion of fibromyalgia patients has not been definitively quantified. However, we are concerned that the generalizability of these results to the FMS patient population treated by pain specialists may be problematic since these patients are typically those who have failed treatment by primary care providers. Also, patients with any psychiatric comorbidity other than isolated MDD, including MDD with coexistent anxiety disorder, were excluded. This would also tend to limit broad applicability of the study results since comorbid psychiatric diagnoses, particularly the anxiety disorders, coexist in approximately $40 \%$ of FMS patients. ${ }^{2}$

\section{Safety and tolerability of duloxetine}

The most concise source for data on the safety and tolerability of duloxetine comes from a meta-analysis of data from eight randomized, double-blind, RCTs on patients with MDD included in the initial New Drug Application to the FDA in the US and the analogous approval process in the European Union..$^{38}$ Overall, $9.7 \%$ (vs $4.2 \%$ of placebo group) of patients in the RCTs discontinued duloxetine because of TEAEs. While these data cannot be directly compared to the FMS trials because of different patient populations, study designs and durations, similar rates of discontinuation were seen in the three duloxetine FMS RCTs. ${ }^{25-27}$ Nausea was the most common TEAE, occurring in $19.9 \%$ of duloxetine-treated subjects and $6.9 \%$ of placebo-treated subjects. Other TEAEs occurring at a higher numeric rate in duloxetine-treated groups included dry mouth, vomiting, decreased appetite, constipation, insomnia, dizziness, fatigue, somnolence, and adverse sexual side effects. Since the different dose groups within each trial were pooled, analysis of dose-dependent side effects from the meta-analysis is limited. However, the FMS trials demonstrated similar TEAEs, with nausea, dry mouth, and constipation being the three most common and occurrences were seen at a numerically higher rate with increased duloxetine dose.

Small but statistically significant changes in weight have been noted with duloxetine treatment. ${ }^{38}$ On average, patients initially lose $0.46 \mathrm{~kg}$ but typically return to baseline weight
12 weeks after initiation of duloxetine followed by weight gain. Weight gain is dose dependent, with the percentage of patients gaining $>7 \%$ of baseline bodyweight numbering $3.1 \%$ on placebo, $8.6 \%$ on duloxetine $60 \mathrm{mg} /$ day, and $12.8 \%$ on $120 \mathrm{mg} /$ day. While the initial weight loss correlates with the period of maximum rates of nausea, vomiting, and decreased appetite, a causal relationship between these TEAEs and weight loss has not been established. Average time to onset for nausea is typically within one day of treatment with a time to resolution averaging seven days in the MDD trials. ${ }^{38}$

As fatigue and sleep disturbance are often significant problems for FMS patients in addition to pain, the side effect profile of duloxetine raises concerns about use in these FMS patients. Sleep disturbance and fatigue trended toward, but did not reach, statistical significance in the Russell et al study, ${ }^{27}$ and a dose-response relationship with somnolence symptoms was seen in duloxetine-treated patients in the second FMS RCT that reached statistical significance in the highest dosage group (60 mg twice daily) ${ }^{26}$ No specific data on the impact of duloxetine on sleep in FMS patients is available. However, there have been investigations performed in patients with $\mathrm{MDD}^{44,45}$ and diabetic peripheral neuropathic pain (DPNP) ${ }^{46}$ Since MDD and DPNP patients frequently experience sleep disturbances and chronic painful physical symptoms as manifestations of their disease, , $^{47}$ conclusions drawn from studies of the effect of duloxetine on sleep in these patients with MDD may have applicability to FMS patients. Polysomnographic studies on subjects with MDD taking $60 \mathrm{mg}$ duloxetine once daily showed changes in stage 3 and rapid eye movement (REM) sleep, including significant increases in Stage 3 sleep duration (from $21.0 \pm 10.7$ to $37.4 \pm 20.1 \mathrm{~min}$ ) and REM latency (from $58.5 \pm 31.1$ to $193.6 \pm 72.6 \mathrm{~min}$ ), and decreased total REM sleep (from $94.8 \pm 34.5$ to $51.5 \pm 42.5 \mathrm{~min}$ ) after 7 to 13 days of treatment with duloxetine (all times expressed as mean \pm standard deviation). ${ }^{45}$ Since restorative sleep occurs during stage 3 and 4 sleep, and FMS patients often have decreased time spent in these sleep stages, these results indicate duloxetine may be beneficial to sleep architecture in FMS patients. Unfortunately, the prolongation of stage 3 sleep was not supported by polysomnographic studies in a group of healthy controls treated with duloxetine that found similar changes in REM sleep. ${ }^{49}$ However, this study was conducted in males and used higher duloxetine doses (both $80 \mathrm{mg}$ once daily and $60 \mathrm{mg}$ twice daily), so it may not be as valid a comparison to FMS patients. Studies on the effect duloxetine has on sleep in FMS patients are needed. 
Since, polysomnographic data does not always correspond to clinically important sleep outcomes, a recent investigation of duloxetine treatment in patients with MDD that evaluated clinically important sleep outcomes may be more relevant. ${ }^{44}$ This study measured sleep disturbance in three insomnia categories including early (sleep onset), middle, and late (early awakening) utilizing the $\mathrm{HAMD}_{17}$ sleep items and spontaneous subjective reports of sleep-related TEAEs. Duloxetine-treated subjects had a statistically significant improvement in early and late onset insomnia but no improvement in middle insomnia. However, the end-average $\mathrm{HAMD}_{17}$ insomnia score for duloxetine-treated subjects was 1.9 vs 2.1 in placebo-treated subjects on a 6-point scale. While this difference reached statistical significance, it is likely not a clinically relevant change. Also, $6.5 \%$ of study subjects took other sleep aid medications, which may have obscured the clinical impact of duloxetine on sleep. It was noted that duloxetine-treated patients had a significantly higher rate of TEAEs such as 'abnormal dreams' and 'hypersomnia' that correlated with these subjects reporting smaller increments of overall sleep improvement. Sleep and depression symptom improvement also correlated, suggesting sleep enhancement may be due to treatment of MDD rather than via a direct beneficial effect of duloxetine on sleep. Pooled data from three studies in DPNP patients without MDD also suggested duloxetine enhances sleep indirectly, as improvement in sleep correlated with reductions in pain based on BPI measurements including ratings of pain interference with sleep. ${ }^{46}$ These results excluded patients with treatment-emergent somnolence, which could be classified as a TEAE. Overall these results are inconclusive for strong evidence for or against duloxetine use in FMS patients with underlying Insomnia symptoms. Patients treated with duloxetine should be informed they may experience sleep-related side effects.

Abnormal laboratory value rates were reported in the previously discussed meta-analysis of duloxetine MDD trials $^{38}$ and the FMS duloxetine RCTs. ${ }^{25-27}$ While there were some small but statistically significant changes noted, these changes were not consistently seen among the trials. Aspartate aminotransferase, alanine aminotransferase, alkaline phosphatase, and creatine phosphokinase levels all rose to a statistically significant degree in at least one of the trials, but the investigators determined these changes to be clinically nonsignificant since the values remained within normal reference ranges. However, the manufacturer recommends against use of duloxetine in patients with hepatic insufficiency or severe alcohol use, ${ }^{37}$ and we recommend baseline measurement of liver enzymes be performed followed by periodic monitoring in all patients treated with duloxetine and that duloxetine treated patients be counseled to avoid alcohol. Statistically significant decreases in serum sodium were noted in the Russell et al study, ${ }^{27}$ but this was deemed clinically nonsignificant based on values remaining within normal reference ranges. Hyponatremia is a recognized side effect of SSRIs ${ }^{50}$ so its occurrence with duloxetine, which has serotonergic activity, is not surprising. Therefore, baseline and periodic monitoring of basic serum chemistries, including sodium, is prudent and recommended.

Anti-depressants and other psychotropic medications are known to be associated with cardiac side effects including QT prolongation with TCAs and sudden cardiac death associated with typical and atypical antipsychotics. ${ }^{51}$ While electrocardiogram (ECG) data were not collected in all of the trials included in the pooled data from initial depression efficacy trials,${ }^{38}$ no significant effect on QT or QTc interval was noted and no ECG effects were seen in the duloxetine FMS trials. ${ }^{25-27}$ Duloxetine administration has consistently been associated with a statistically significant increase in heart rate, systolic and diastolic blood pressure in both $\mathrm{MDD}^{31}$ and FMS trials, ${ }^{25-27}$ but the average increase has been uniformly small and not deemed clinically significant when considering the data as a whole by any of the investigators. However, the actual clinical effect in individual patients, especially those with comorbid hypertension or heart disease, cannot be predicted based on aggregate data and baseline and periodic monitoring of blood pressure and heart rate is recommended. ${ }^{37}$

Also, concerns that increased circulating norepinephrine levels induced by duloxetine could negatively affect cardiac function and induce harmful cardiac remodeling have been voiced, ${ }^{52}$ implying that duloxetine treatment may negatively impact patients with pre-existing congestive heart failure (CHF). This concern is supported by case reports of worsening heart failure after initiating treatment with venlafaxine ${ }^{53}$ and duloxetine. ${ }^{52}$ No prospective studies have evaluated the effect of duloxetine treatment in patients with CHF.

In addition to possible cardiac side effects, duloxetine treatment also presents a theoretical bleeding risk since serotonin-active medications are known to decrease platelet aggregation, ${ }^{54}$ and a retrospective data review found a statistically significant increased risk of gastrointestinal bleeding when SSRIs were used in combination with nonsteroidal anti-inflammatory medications (NSAIDs). ${ }^{55}$ While pooled data from trials of duloxetine in MDD have found no statistically significant increase in bleeding 
disorders compared to placebo, ${ }^{38}$ NSAID use is common in FMS patients. Since the effect on bleeding risk in individual patients cannot be predicted, caution is advised when considering duloxetine use in patients with a history of gastrointestinal (GI) bleeding or patients taking NSAIDs. Concomitant proton pump inhibitor treatment should be considered in patients at risk for bleeding complications.

The FDA requires that antidepressant medications, including duloxetine, contain a 'black box warning' in their prescribing information indicating use may increase the risk of suicidality. This is because some patients with MDD and other psychiatric disorders have developed suicidal ideation upon initiating anti-depressant medication therapy. This has been theoretically attributed to the stimulating properties of these medications that may lead patients to experience an increase in energy and motivation before there is resolution of other depressive symptoms such as suicidal thoughts. Suicide risk is most prominent in patients aged 24 years old or younger with 14 additional episodes of suicidality per 1000 patients compared to placebo in patients less than 18 years of age and 5 additional cases in patients aged 18 to 24 years. ${ }^{37}$ Data imply a more neutral profile for patients 25 to 64 years of age, with 1 fewer case for each 1000 patients treated, and protective effects were noted in patients 65 years and older with 6 fewer cases. However, since individual suicidal risk cannot be predicted, all patients should be warned about the possible increased risk of suicidality and encouraged to seek emergency care if suicidal thoughts occur.

Discontinuation of duloxetine has been associated with withdrawal symptoms, including dizziness, nausea, and headache in patients who have received the medication to treat depression. ${ }^{37}$ If duloxetine must be discontinued, a slow down-titration taper is recommended to minimize withdrawal symptoms. If patients experience clinically significant symptoms with dose taper, the dosage should be increased and then tapered more slowly. ${ }^{37}$

\section{Patient focused perspectives on use of duloxetine in FMS management}

Reviewing the three duloxetine FMS treatment trials to estimate medication tolerability, ${ }^{25-27}$ the highest adherence rate in the trials was seen in the $60 \mathrm{mg}$ /day duloxetine treatment group in the 2005 Arnold et al study, ${ }^{26}$ with $65 \%$ of patients completing the 12-week trial. In this RCT, more patients in the duloxetine treatment groups completed the trial compared with placebo, and more patients treated with placebo discontinued the study due to lack of efficacy than those treated with duloxetine. This result was statistically significant suggesting, not surprisingly, that patients with symptom improvement were more likely to continue taking duloxetine. Consistent with this result, duloxetine-treated patients in the Russell et al study ${ }^{27}$ were more likely to complete the trial compared with placebo-treated patients. In contrast, placebo-treated patients in the 2004 Arnold et al trial ${ }^{25}$ were more likely to be compliant than those on active drug (64\% vs 56\%, respectively). The data indicates treatment adherence can be increased by dosing strategies that maximize both efficacy and tolerability. Since TEAEs are known to increase with duloxetine dose, and therapeutic efficacy has been seen in patients treated with duloxetine $20 \mathrm{mg}$ once daily, ${ }^{27}$ the authors recommend FMS patients be started on duloxetine at a dose of $20 \mathrm{mg}$ once daily where available and monitored for treatment response for at least 1 week before the dose is increased. If patients fail to respond, the dose should be increased in $20 \mathrm{mg}$ intervals and patient response evaluated before increasing. Because of the greater incidence of TEAEs with duloxetine at doses of $120 \mathrm{mg} /$ day and the lack of improved efficacy compared with duloxetine $60 \mathrm{mg} /$ day, ${ }^{26}$ patients with FMS should not be treated with doses exceeding $60 \mathrm{mg} /$ day to reduce the likelihood of discontinuation secondary to adverse events.

Based on data from the three duloxetine FMS RCTs and duloxetine's FDA indications for the management of both depression and anxiety, ${ }^{25-27,37}$ duloxetine is most likely to benefit middle-aged females with FIBRO symptoms of Blues (mood disorders) and Ow! (pain). Furthermore, in the Russell et al study, ${ }^{27}$ all doses of duloxetine significantly improved attention and concentration (as measured by the MFI mental fatigue domain) suggesting FMS patients with cognitive dysfunction ('fibrofog') may benefit from duloxetine treatment. Patients with FMS are known to have Irritable Bowel Syndrome (IBS) with prevalence rates estimated between $32 \%$ to $80 \% .{ }^{56,57}$ Because the FMS RCTs show GI symptoms such as nausea, constipation and decreased appetite were more likely to occur in FMS patients treated with duloxetine compared with placebo, ${ }^{25-27}$ clinicians must use duloxetine with caution in FMS patients with IBS. In these individuals, it is especially important to use the "start low and go slow" approach with a $20 \mathrm{mg} /$ day dose used first since it appears to have the lowest occurrence of GI symptoms. ${ }^{27}$

FMS patients with FIBRO symptoms of Fatigue and Insomnia should be warned about the potential for duloxetine to exacerbate their symptoms, as insomnia occurred more frequently in patients treated with duloxetine 
compared with placebo. ${ }^{27,37}$ Furthermore, somnolence in all duloxetine-treated groups occurred at twice the rate of placebo in the Russell et al study, ${ }^{27}$ which may exacerbate Fatigue symptoms. However, it is important to remember that the impact of duloxetine on sleep in FMS patients treated with duloxetine has not been thoroughly investigated.

\section{Conclusions and key points}

- FMS is a complex disorder best managed utilizing an individualized therapeutic regimen that includes both pharmacologic and nonpharmacologic treatments that addresses the entirety of patient symptoms.

- The FIBRO mnemonic can be used to recall FMS symptoms including Fatigue, Insomnia, Blues, Rigidity, and Ow! for pain.

- Primary disorders that can mimic FMS must be ruled out before instituting symptomatic therapies.

- Pharmacologic treatments should utilize a 'start low and go slow' approach.

- Duloxetine is an SNRI medication indicated for the management of adult FMS patients at a dose of $60 \mathrm{mg}$ once daily, however, lower doses may be effective and we recommend starting with a $20 \mathrm{mg}$ once daily dose with increases in weekly intervals only if needed.

- The pain ameliorating effect of duloxetine is independent of its effect on mood, however, since it is the only FMS medication also indicated to treat MDD and anxiety syndrome duloxetine should be the first line therapy in FMS patients with coexisting mood disorders.

- Duloxetine may be particularly beneficial in FMS patients with significant cognitive dysfunction ('fibrofog'), but it may worsen symptoms of Fatigue and Insomnia.

- TEAEs seen in duloxetine trials include nausea, dry mouth, vomiting, decreased appetite, constipation, insomnia, dizziness, fatigue, somnolence, and adverse sexual side effects.

- Duloxetine may increase suicide risk, particularly in patients 24 years and younger, and use is not recommended in those $<18$ years of age.

- Duloxetine use has been associated with liver function test abnormalities and hyponatremia, therefore, baseline and surveillance laboratory monitoring are recommended and patients should be counseled to avoid alcohol use.

- Duloxetine should be used with caution in patients with pre-existing cardiovascular disease and/or increased bleeding risk.

\section{Disclosure}

CSB is on the speaker's bureau for Eli-Lilly Inc., Pfizer Inc., and Forest pharmaceuticals Inc. and receives support from Pfizer Inc. and the National Institutes of Health.

\section{References}

1. Wolfe F, et al. The American College of Rheumatology. Criteria for the classification of fibromyalgia. Arthritis Rheum. 1990;33:160-172.

2. Neumann L, Buskila D. Epidemiology of fibromyalgia. Curr Pain Headache Rep. 2003;7:362-368.

3. Katz RS, et al. Fibromyalgia Diagnosis: a comparison of clinical, survey, and American College of Rheumatology criteria. Arthritis Rheum. 2006;54(1):169-176.

4. Berenson A. Drug approved. Is disease real? http://www.nytimes. com/2008/01/14/health/14pain.html?scp=2\&sq=fibromyalgia\&st=cse. Accessed June 6, 2009.

5. Powers R. Fibromyalgia: an age-old malady begging for respect. J Gen Intern Med. 1993;8(2):93-105.

6. White KP, Thompson J. Fibromyalgia syndrome in an Amish community: A controlled study to determine disease and symptom prevalence. $J$ Rheum. 2003;30(8):1835-1840.

7. Ablin JN, et al. Mechanisms of disease: genetics of fibromyalgia. Nature Clin Pract Rheum. 2006;2(12):671-678.

8. Dadabhoy D, et al. Biology and therapy of fibromyalgia. Evidencebased biomarkers for fibromyalgia syndrome. Arthritis Res Ther. 2008;10(4):211-228.

9. Annemans L, et al. Health economic consequences related to the diagnosis of fibromyalgia syndrome. Arthritis Rheum. 2008;58(3):895-902.

10. Carville SF, et al. EULAR evidence-based recommendations for the management of fibromyalgia syndrome. Ann Rheum Dis. 2008;67: 536-541.

11. Boomershine CS, Crofford LJ. A symptom-based approach to pharmacologic mangement of fibromyalgia. Nat Rev Rheumatol. 2009;5(4):191-199.

12. Boomershine CS, et al. Five visual analogue scales quantify global disease severity and identify clinically significant symptoms in fibromyalgia syndrome. Arthritis Rheum. 2008;58:s686-s687.

13. Mease P. Fibromyalgia syndrome: review of clinical presentation, pathogenesis, outcome measures, and treatment. J Rheum. 2005; 32(Suppl 75):6-21.

14. Slotkoff AT, et al. The relationship between fibromyalgia and the multiple chemical sensitivity syndrome. Scand J Rheum. 1997;26: 364-367.

15. Sharma J, et al. Pharmakokinetics and safety of duloxetine, a dualserotonin and norepinephrine reuptake inhibitor. J Clin Pharmacol. 2000;40(2):161-167.

16. Trivedi MH, et al. Clinical evidence for serotonin and norepinephrine reuptake inhibition of duloxetine. Int Clin Psychopharmacol. 2008;23(3):161-169.

17. Schatzberg AF. Safety and tolerability of antidepressants: weighing the impact on treatment decisions. J Clin Psychiatry. 2007;68(Suppl 8): $26-34$.

18. Stahl SM, et al. SNRIs: Their pharmacology, clinical efficacy, and tolerability in comparison with other classes of antidepressants. CNS Spectr. 2005;10(9):732-747.

19. Deecher DC, et al. Desvenlafaxine succinate: a new serotonin and norepinephrine reuptake inhibitor. J Pharmacol Exp Ther. 2006;318(2):657-665.

20. Owens MJ, et al. Second-generation SSRIs: human monoamine transporter binding profile of escitalopram and $R$-fluoxetine. Biol Psychiatry. 2001;50:345-350.

21. Westanmo AD, et al. Duloxetine: a balanced and selective norepinephrineand serotonin-reuptake inhibitor. Am J Health Syst Pharm. 2005;62(23): 2481-2490. 
22. Fric M, et al. The influence of smoking on the serum level of duloxetine. Pharmacopsychiatry. 2008;41(4):151-155.

23. Preskorn J. Duloxetine. J Psychiatr Pract. 2004;10(6):375-385.

24. Wells KA, Losin WG. In vitro stability, potency, and dissolution of duloxetine enteric-coated pellets after exposure to applesauce, apple juice, and chocolate pudding. Clin Ther. 2008;30(7):1300-1308.

25. Arnold LM, et al. A double-blind, multicenter trial comparing duloxetine with placebo in the treatment of fibromyalgia patients with or without major depressive disorder. Arth Rheum. 2004;50(9):2974-2984.

26. Arnold LM, et al. A randomized, double-blind, placebo-controlled trial of duloxetine in the treatment of women with fibromyalgia with or without major depressive disorder. Pain. 2005;119(1-3):5-15.

27. Russell IJ. Efficacy and safety of duloxetine for treatment of fibromyalgia in patients with or without major depressive disorder: Results from a 6-month, randomized, double-blind, placebo-controlled, fixed-dose trial. Pain. 2008;136(3):432-444.

28. Bennett R. The Fibromyalgia Impact Questionnaire (FIQ): a review of its development, current version, operating-characteristics, and uses. Clin Exp Rheum. 2005;23(Suppl 39):S154-S162.

29. Cleeland CS, Ryan KM. Pain assessment: global use of the brief pain inventory. Ann Acad Med Singapore. 1994;23(2):129-138.

30. Guy W. ECDEU assessment manual for psychopharmacology, revised. Rockville, MD: US Department of Health, Education, and Welfare publication (ADM). National Institute of Mental Health; 1976. p. 76-338.

31. Beck AT, et al. Comparison of Beck Depression Inventories -IA and -II in psychiatric outpatients. J Pers Assess. 1996;67:588-597.

32. Beck AT, Epstein N, Brown G, Steer RA. An inventory for measuring clinical anxiety: psychometric properties. J Consult Clin Psychol. 1988;56:893-897.

33. Ware JE, Snow KK, Kosinski M, et al. SF-36 health survey manual and interpretation guide. Boston (MA): The Health Institute, New England Medical Center; 1993.

34. Sheehan DV, et al. The measurement of disability. Int Clin Psychopharmacol. 1996;11(Suppl 3):89-95.

35. Wolfe F, et al. Aspects of fibromyalgia in the general population: sex, pain threshold, and fibromyalgia syndrome. J Rheum. 1995;22(1):151-156.

36. Retherford RD, Choe MK. Statistical methods for causal analysis. New York: John Wiley \& Sons; 1993.

37. http://pi.lilly.com/us/cymbalta-pi.pdf. Accessed May 1, 2009.

38. Hudson JI, et al. Safety and tolerability of duloxetine in the treatment of major depressive disorder: analysis of pooled data from eight placebo-controlled clinical trials. Hum Psychopharmacol. 2005;20(5): 327-41.

39. Hamilton M. A rating scale for depression. J Neurol Neurosurg Psychiatry. 1960;23:56-62.

40. Fischer AA. Pressure threshold meter: its use for quantification of tender spots. Arch Phys Med Rehabil. 1986;67(11):836-838.

41. Dadabhoy D, et al. Evidence-based biomarkers for fibromyalgia syndrome. Arth Res. Ther. 2008;10(4):211.
42. McCarberg B, et al. Tender points as predictors of distress and the pharmacologic management of fibromyalgia syndrome. Am J Ther. 2003;10(3):176-192.

43. Smets EM, et al. The Multidimensional Fatigue Inventory (MFI) psychometric qualities of an instrument to assess fatigue. J Psychosom Res. 1995;39:315-325.

44. Brecht S, et al. Clinical impact of duloxetine treatment on sleep in patients with major depressive disorder. Int Clin Psychopharmacol. 2008;23(6):317-324.

45. Kluge M, et al. Duloxetine increases stage 3 sleep and suppresses rapid eye movement (REM) sleep in patients with major depression. Eur Neuropsychopharmacol. 2007;17(8):527-531.

46. Fishbain DA, et al. Does pain mediate the pain interference with sleep problem in chronic pain? Findings from studies for management of diabetic peripheral neuropathic pain with duloxetine. J Pain Symptom Manage. 2008;36(6):639-647.

47. Culpepper L. Secondary insomnia in the primary care setting: review of diagnosis, treatment, and management. Curr Med Res Opin. 2006;22(7):1257-1268.

48. Ohayon MM, Schatzberg AF. Using chronic pain to predict depressive morbidity in the general population. Arch Gen Psychiatry. 2003;60(1):39-47.

49. Chalon $\mathrm{S}$, et al. Comparative effects of duloxetine and desipramine on sleep EEG in healthy subjects. Psychopharmacology (Berl). 2005;177(4):357-365.

50. Wright SK, Schroeter S. Hyponatremia as a complication of selective serotonin reuptake inhibitors. J Am Acad Nurse Pract. 2008;20(1): 47-51.

51. Ray WA, et al. Atypical antipsychotic drugs and the risk of sudden cardiac death. $N$ Engl J Med. 2009;360(3):225-235.

52. Colucci VJ. Heart failure worsening and exacerbation after venlafaxine and duloxetine therapy. Ann Pharmacother. 2008;42(6):882-887.

53. Drent $\mathrm{M}$, et al. Drug-induced pneumonitis and heart failure simultaneously associated with venlafaxine. Am J Respir Crit Care Med. 2003;167(7):958-961.

54. de Abajo FJ, et al. Association between selective serotonin reuptake inhibitors and upper gastrointestinal bleeding: population based casecontrol study. Brit Med J. 1999;319(7217):1106-1109.

55. Mort JR, et al. Interaction between selective serotonin reuptake inhibitors and nonsteroidal antiinflammatory drugs: review of the literature. Pharmacotherapy. 2006;26(9):1307-1313.

56. Sperber AD, et al. Fibromyalgia in the irritable bowel syndrome: studies of prevalence and clinical implications. Am J Gastroenterol. 1999;94:3543-3546.

57. Kurland JE, et al. Prevalence of irritable bowel syndrome and depression in fibromyalgia. Dig Dis Sci. 2006;51:454-460.

58. Brunton LL, editor. Potencies of antidepressants at human transporters for monoamine neurotransmitters. Goodman \& Gillman's The Pharmacological Basis of Therapeutics, 11 th ed. New York: McGrawHill; 2006.
Journal of Pain Research

\section{Publish your work in this journal}

The Journal of Pain Research is an international, peer-reviewed, open access, online journal that welcomes laboratory and clinical findings in the fields of pain research and the prevention and management of pain. Original research, reviews, symposium reports, hypothesis formation and commentaries are all considered for publication.

\section{Dovepress}

The manuscript management system is completely online and includes a very quick and fair peer-review system, which is all easy to use. Visit http://www.dovepress.com/testimonials.php to read real quotes from published authors. 